\title{
Erasmus syndrome in a marble worker
}

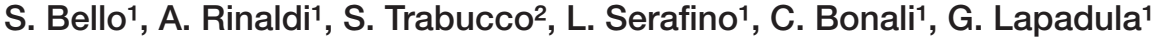 \\ ${ }^{1}$ Rheumatology Unit, Policlinico Hospital, University of Bari, Bari, Italy; '2Department of Pathological Anatomy, \\ Policlinico Hospital, University of Bari, Bari, Italy
}

\section{SUMMARY}

Erasmus syndrome is defined as the association of silica exposure and subsequent development of systemic sclerosis. The limited number of cases reported in the literature mainly involves miners and only sporadically other professionals. We describe a case of Erasmus syndrome in a marble worker.

A 68 year old man came to our observation complaining pelvic and scapular girdle pain, evening fever, intense weakness and emaciation for about 1 month. He also reported to have had Raynaud's phenomenon in his hands for the last 13 years. Also, his occupational history revealed a chronic exposure to silica dust. The patient presented pain in his shoulders and hips, moderate skin thickening and sclerosis in his hands and fingers extending proximally to his wrists. The diagnosis of systemic sclerosis was determined according to his clinical and medical history, the positivity of anti-Scl 70 antibodies, the nailfold capillaroscopy suggestive of an active scleroderma pattern and the detection of a mild restrictive pulmonary syndrome. The evaluation of the organbased complications excluded a gastroenterological and cardiovascular involvement, while the chest computed tomography (CT) detected multiple small nodules with a mantle distribution and enlarged lymph nodes with no signs of interstitial lung disease and fibrosis. Additional tests (positron emission tomography-CT, flexible bronchoscopy and broncho-alveolar lavage) excluded infectious diseases and cancer. However, given the pulmonary involvement, we performed a histological examination of the parenchyma and lymph nodes, which revealed a picture of pneumoconiosis. In the end, the occupational history and the findings from the diagnostic procedures led to the diagnosis of pulmonary silicosis. The precise definition of the pulmonary involvement was essential to the therapeutic approach to this patient.

Key words: Erasmus syndrome; systemic sclerosis; silicosis; pneumoconiosis.

Reumatismo, 2015; 67 (3): 116-122

\section{INTRODUCTION}

$\mathrm{T}$ he Erasmus syndrome is characterized by the association of exposure to silica with or without silicosis and the subsequent development of systemic sclerosis (SSc). This syndrome is named after the doctor who first described 17 cases of SSc in gold miners in South Africa in 1957 (1). Subsequently, Rodnan et al. reported that, in a series of 150 cases of SSc, 26 of the 60 males $(43 \%)$ had a history of long occupational exposure to silica dust (2). A subsequent meta-analysis of 16 studies on this subject suggested that exposure to silica is a significant risk factor in the development of SSc, particularly in males (3). Not only is exposure to silica known to be responsible for silicosis, but also for other auto- immune diseases. In a French study of 764 patients hospitalized for connective tissue disease (CTD) over the course of 10 years, 24 patients $(3 \%)$ reported a history of occupational exposure to silica.

They were predominantly males, immigrants, miners and sandblasters. The prevalence of SSc was significantly higher in this group than in patients with CTD not associated with silica exposure (4).

In a retrospective study of patients with silicosis in the State of Michigan, the prevalence of rheumatoid arthritis was $4.2 \%$, that of SSc and Sjögren's syndrome was $0.3 \%$, that of systemic lupus erythematosus was $0.1 \%$ and that of anti-neutrophil cytoplasmic antibody (ANCA)-associated vasculitis was $0.8 \%$. The risk of SSc appeared to be 24 times greater in patients 
with silicosis than in the general population (5).

According to the most accredited theory that explains the association between chronic exposure to silica and autoimmune disorders, this process is triggered by the dysregulation of the apoptosis in lymphocytes. Central is the role of Fas, a protein belonging to the tumor necrosis factor receptor family and mainly expressed on the cell membrane of lymphocytes. Its interaction with the Fas ligand triggers the caspase cascade that leads to DNA fragmentation $(6,7)$. Patients with silicosis show an increased expression of anti-apoptotic substances in the peripheral blood mononuclear cells, such as soluble Fas and DcR3 gene expression protein, as well as a lower expression of membrane Fas $(8,9)$. It was also noted that the percentage of CD4+d, $\mathrm{CD} 25+$, FoxP3+, or regulatory $\mathrm{T}$ cells (Treg) with suppressor activity on excess auto-reaction, besides being slightly reduced in patients with silicosis, also perform a less efficient function in these patients compared with healthy volunteers (10).

Therefore, the inflammatory response to chronic exposure to silica triggers the $\mathrm{T}$ lymphocyte polyclonal activation and the formation of T-lymphocyte auto reactive clones, refractory to the apoptosis process. This, in turn, leads to the appearance of auto-antibodies and the development of the autoimmune disease (11). Various studies demonstrated, indeed, the presence of auto-antibodies in patients exposed to silica dust. In a study of 61 subjects with a history of occupational exposure to silica and without a known rheumatologic disease, $11.4 \%$ were positive for rheumatoid factor (RF), $1.6 \%$ was positive for the antinuclear antibody (ANA) and $1.6 \%$ had ANCA. All RF-positive patients had pulmonary silicosis and the positivity for RF correlated with their long exposure to silica dust (12). In a study of 14 patients with silica-associated SSc, 9 patients had antibodies against topo-isomerase I, and 1 patient had anticentromere antibodies (13). In a study of 22 miners with a long exposure to silica dust, 30 miners with silicosis and 17 miners with
SSc associated with silica exposure, ANA were positive in $13.6 \%$ of the exposed patients, in $46.7 \%$ of the patients with pulmonary silicosis and in $94.1 \%$ of patients with Erasmus syndrome. In the third group, $47.1 \%$ of patients were also positive for anti-Scl70 antibodies. The same study reported that patients with Erasmus syndrome had indistinguishable characteristics from patients with idiopathic SSc. They all had Raynaud's phenomenon and skin sclerosis (14 limited and 3 generalized). Ten of them had necrosis of the fingertips, 16 pulmonary fibrosis and 9 esophageal involvement (14). Only few other cases of Erasmus syndrome are described in the literature (1522). Besides affecting workers in the gold (1) and coal $(2,14)$ mines, Erasmus syndrome was also described in other professionals, such as sculptors and individuals exposed to abrasive powders (4), drillers (17), quartz crushing worker (22) and dental technicians $(20,21)$. We report a case of Erasmus syndrome in a patient who had worked for 46 years cutting and finishing granite and quartz slabs.

\section{CASE REPORT}

A 68 year old male came to our observation due to the onset of fatigue, evening fever, weight loss, pelvic and scapular girdle inflammatory pain for about 1 month. Since he was 55, he had had Raynaud's phenomenon in the hands, which occurred more frequently in the last year. He did not report other symptoms, such as dyspnea and dysphagia. He was treated for essential hypertension for an undefined period of time and reported to have worked as marble worker for about 46 years.

The patient presented pain in his shoulders and hips, no apparent functional deficits during mobilization, moderate skin thickening, sclerosis of fingers and hands extending proximal to his wrists (modified Rodnan skin score 9/51; Figure 1) and the presence of ulcerative lesions in the perimalleolar region of his legs. No abnormal findings were reported in the diagnostic investigations: blood counts, liver and kidney function tests, metabolic investigations, 


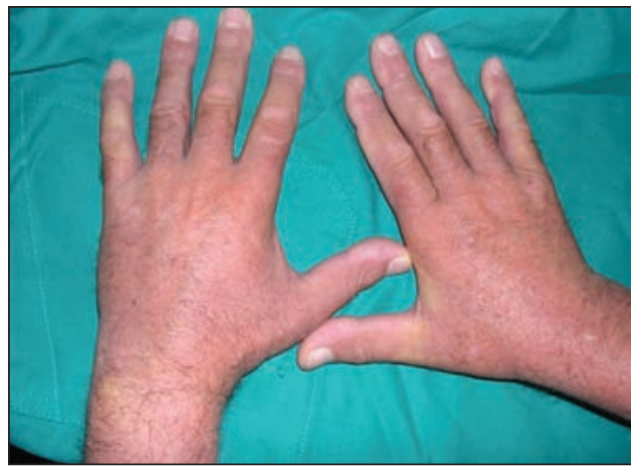

Figure 1 - Moderate skin thickening and sclerosis of the fingers and the hands extending proximally to the wrists.

thyroid function, phosphocalcic metabolism, tumor markers (AFP, CEA, CA15.3, CA125, CA 19.9, PSA), B and C hepatitis virus markers, urine test, $\mathrm{C} 3$ and $\mathrm{C} 4$ fraction of complement and immunoglobulins level. Also RF, cryoglobulins, anti-ds DNA antibodies, anti-CCP antibodies, ACA, anti-MPO and anti-PR3 antibodies tested negative. The acute phase reactants (erythrocyte sedimentation rate $21 \mathrm{~mm}$; C-reactive protein $4.3 \mathrm{mg} / \mathrm{L}, \alpha 2$ globulin $15.1 \%$ )

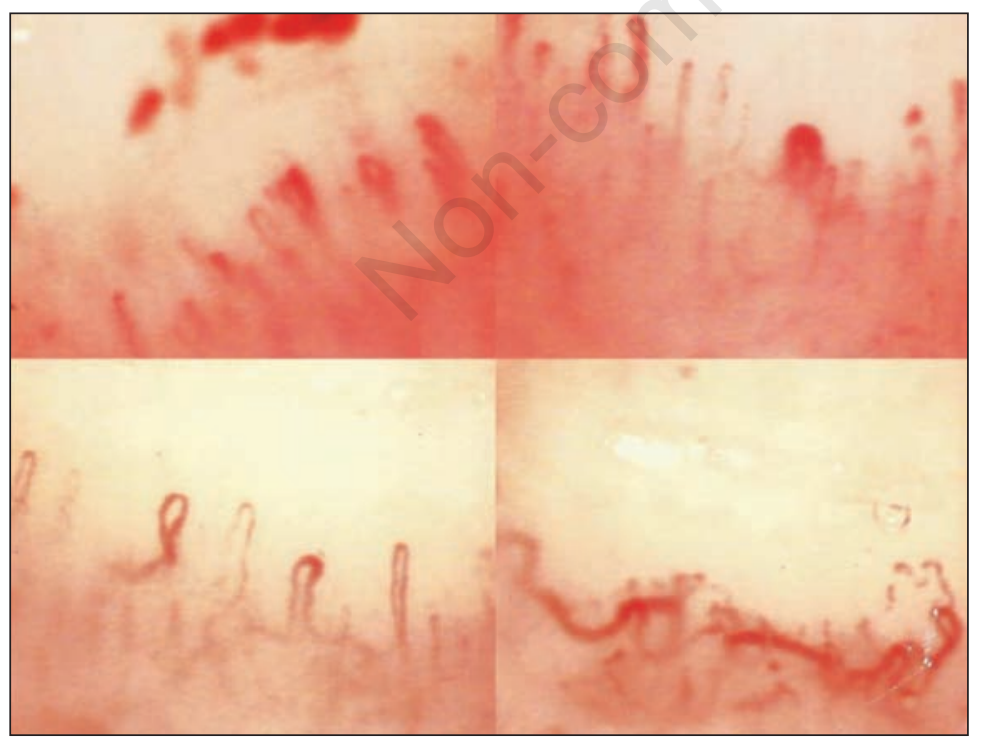

Figure 2 - Nailfold capillaroscopy. Simultaneous presence of numerous abnormalities typical of the active scleroderma pattern: hemorrhages, giant capillaries, edema, vascular architectural disorganization and avascular areas. and the lactate dehydrogenase assay (327 U/L) showed slightly increased values. ANA (1/1280 speckled pattern) and antiScl $70(638.3 \mathrm{U} / \mathrm{mL})$ antibody assays and the quantiferon TB-Gold test were positive. As to the other diagnostic procedures, electrocardiogram, bone densitometry (dualenergy x-ray absorptiometry at the proximal femur), ultrasonography of abdomen, salivary glands, thyroid, shoulders, hands and wrists were normal.

Chest radiography showed signs of chronic obstructive pulmonary disease, an enlargement of the left cardiac section and a mild aortic arch ectasia. The pulmonary function tests showed a reduction of lung volumes, a slight reduction of ventilatory flows, mild restrictive pneumopathy and a moderate decrease of the diffusing capacity of the lung for carbon monoxide (forced vital capacity was $74 \%$, forced expiratory volume in 1 second was $82.2 \%$, lung transfer factor for carbon monoxide was 58\%). Electromyography showed signs of motor and sensory injury (predominantly axonal) in the upper limbs. Nailfold capillaroscopy showed numerous abnormalities typical of the active scleroderma pattern (vascular architectural disorganization, changes in blood flow, edema, hemorrhages, giant capillaries, loss of capillaries and avascular areas; Figure 2). The evaluation of organ-based complications showed neither a gastroenterological involvement (esophageal transit scintigraphy was normal; gastroscopy reveled hyperemia of the gastric mucosa) nor a cardiovascular involvement (six-minute walk test: $521 \mathrm{~m}$; SpO2 98\% stable; echocardiographic pulmonary artery pressures estimation $35 \mathrm{mmHg}$; proBNP dosage $256 \mathrm{ng} / \mathrm{L})$. Chest computed tomography (CT) revealed multiple micronodular formations, predominantly with a mantle distribution, visible in both lungs (the most voluminous, $9.7 \mathrm{~mm}$ of diameter, was in the back basal segment of the lower left lung lobe), and multiple enlarged lymph nodes in the mediastinum (the most voluminous, $15 \mathrm{~mm}$ of diameter, was in the Barety space; Figure 3 ). Chest CT required further pneumological investigations, despite the absence of signs of interstitial 
fibrosis. Arterial blood gas test $(\mathrm{pH}$ : 7.5; pCO2: $40 \mathrm{mmHg}$; pO2: $97 \mathrm{mmHg}$ ), flexible bronchoscopy and bronchoalveolar lavage (bacteria, fungi and $\mathrm{BK}$ tests and the cytological study) were all negative. The positron emission tomography with 2-deoxy-2-[fluorine-18]fluoro- D-glucose integrated with CT (18F-FDG PET/CT) scanning showed radiotracer hypermetabolic areas in the pulmonary hilum bilaterally [standardized uptake value (SUV) max 2.6], in the carinal space (SUV max 2.4), in the aortopulmonary window (SUV max 3 ) and in the Barety space (SUV max 1.5), attributable to lymphadenopathy. No other areas of altered glucose metabolism were found in the remaining anatomical structures examined (Fig. 4).

The histology of the lung parenchymal biopsy reported the presence of multiple interstitial and well-demarcated nodules with a diameter smaller than $1 \mathrm{~cm}$. Some of these modules exclusively consisted of pigmented histiocytes and rare lymphocytes, while others had a sclero-hyaline central area surrounded by a range of pigmented histiocytes and others appeared mainly to be only sclero-hyaline nodules. In the remaining parenchyma, no interstitial fibrosis was found. Numerous converging sclero-hyaline nodules were highlighted in a satellite lymph node. These morphological findings were compatible with the diagnosis of pneumoconiosis (Fig. 5). On the basis of a detailed work history and laboratory and instrumental examinations, the occupational medicine specialist diagnosed pulmonary silicosis.

\section{DISCUSSION}

Silicosis is a pneumoconiosis caused by the inhalation of silicon dioxide powder in the crystalline state or silicate, which results in progressive lung fibrosis. Silicosis can remain latent for several years. The severity of symptoms, such as cough, dyspnea, respiratory and heart failure, depends on the time of exposure to silica dust and the degree of structural impairment of the lung parenchyma. The most common causes of death are respiratory failure, pulmonary hypertension, right heart failure and tuberculosis.

Although this case is similar to others reported in the literature, it presented some peculiarities that made the diagnostic pro-

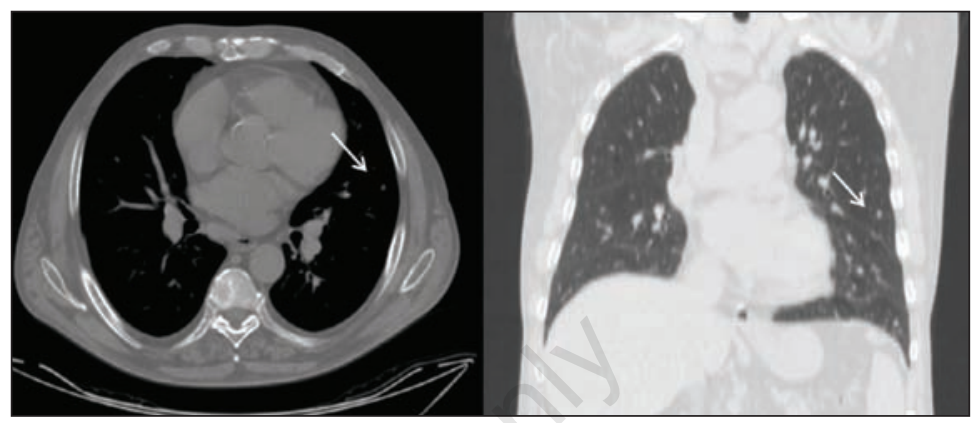

Figure 3 - Chest computed tomography. Presence of multiple micronodular formations, predominantly with a mantle distribution (arrows) and presence of multiple enlarged lymphnodes in the mediastinum.

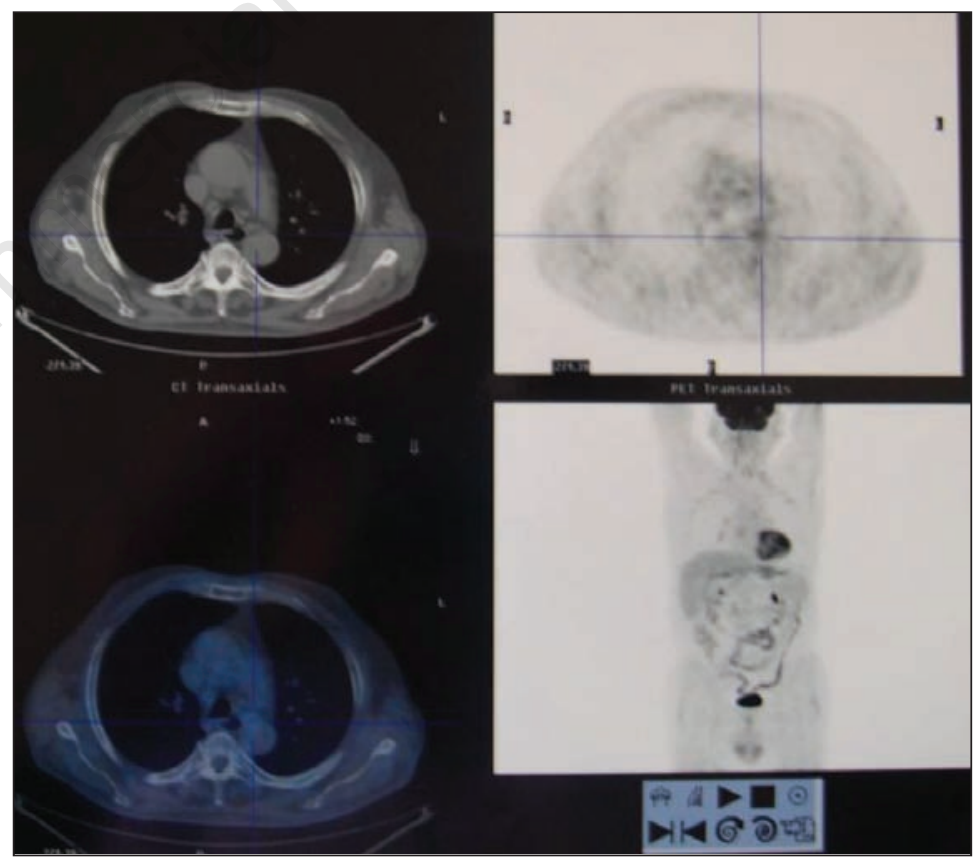

Figure 4 - Positron emission tomography with 2-deoxy-2-[fluorine-18]fluoro-D-glucose integrated with computed tomography scanning. Radiotracer hypermetabolic areas in the pulmonary hilum bilaterally, in the carinal space, in the aortopulmonary window and in the Barety space, attributable to lymphadenopathy and absence of other areas of altered glucose metabolism in the remaining examined anatomical structures. 


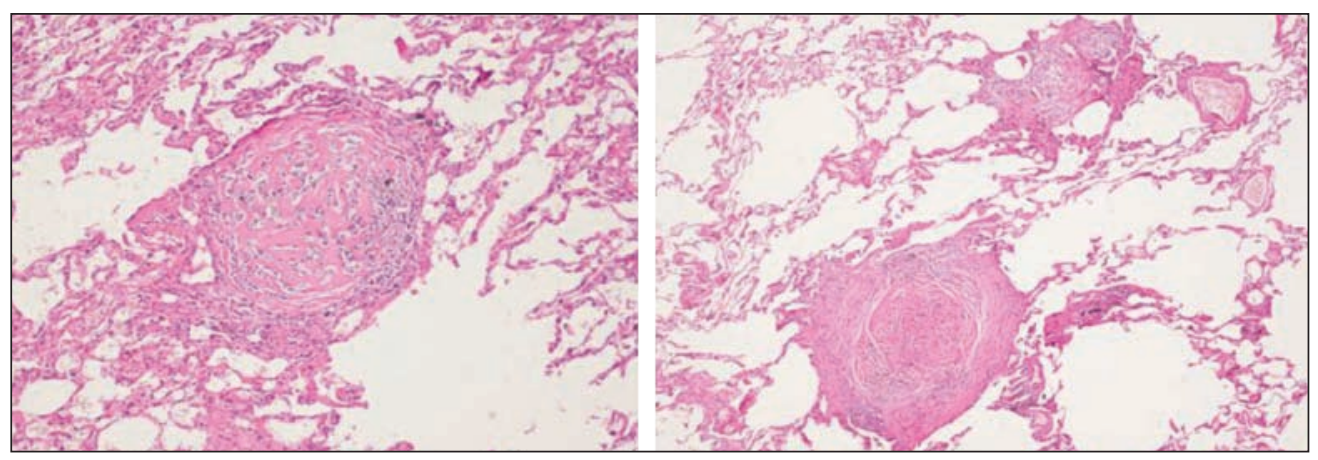

Figure 5 - Lung parenchymal histological examination. Presence of multiple interstitial and well demarcated nodules, some of which consisted of a sclero-hyaline central area surrounded by a range of pigmented histiocytes (picture on the left) and others who presented themselves with predominantly sclero-hyaline nodules (picture on the right).

cess challenging. Erasmus syndrome is by definition characterized by the association of exposure to silica, with or without silicosis, and the subsequent development of SSc (23). Although the exposure to silica was definitely confirmed by the work history and SSc was diagnosed according to the current classification criteria (24), a differential diagnosis was necessary given the presence of a polymyalgia-like onset, systemic impairment, patient sex and age. In particular the diagnostic hypothesis of polymyalgia rheumatica, suggested by the pelvic and scapular girdle pain associated with significant morning stiffness, patient age, fever, fatigue and weight loss, as well as a prompt response to the steroid therapy, was ruled out in favor of a connective tissue disease given the prevailing orientation of the clinical, biochemical and instrumental findings.

A potential diagnosis of tuberculosis was also considered, despite the absence of pulmonary symptoms, due to the systemic onset marked by fever, fatigue and weight loss, positivity of quantiferon TB Gold test and the detection of multiple micronodular parenchymal formations and multiple enlarged lymph nodes in the mediastinum in the chest CT. However, this diagnosis was ruled out because of the lack of altered glucose metabolism in the pulmonary nodules in the PET-CT, the negativity of the bronchoalveolar lavage culture tests and the histological examination of the parenchyma and lymph nodes. The diagnosis of sarcoidosis seemed to be justified by the inflammatory signs at the onset and the detection of mediastinal lymphadenitis in the chest CT, however it was compatible with the sex and age of the patient, the absence of respiratory symptoms, such as cough, dyspnea and hemoptysis, the absence of pulmonary infiltrates in the chest X-ray and $\mathrm{CT}$ and the histological examination. The tentative diagnosis of cancer and paraneoplastic syndrome, suggested by the patient age, weight loss and asthenia and the CT findings of pulmonary nodules and mediastinal lymphadenitis, was ruled out given the negativity of heteroplastic markers, flexible bronchoscopy and the cytological study on the bronchoalveolar lavage, the absence of altered glucose metabolism areas in the various anatomical regions examined in the total body PET-CT, except for the mediastinal lymphnodes, and the histological examination of the parenchyma and lymph nodes.

Finally, only the histology excluded completely the possibility of scleroderma lung disease and confirmed the diagnosis of pulmonary silicosis. This factor was crucial in the therapeutic process. Despite the lack of a gold standard treatment, pulmonary involvement in the course of SSc requires an immunosuppressive therapy, which is reported to be the most appropriate (25). In particular, since the loss of lung function usually occurs in the first 10 years of disease, high-risk patients should begin early therapy with cyclophosphamide, as it can 
delay lung injury progression and thereby increase the patient survival (26). On the contrary, no benefit is reported from any treatment for silicosis and long-term steroid therapy, therefore the only therapeutic solution is to avoid exposure to silica dust (27).

The therapy included methotrexate $15 \mathrm{mg} /$ week, 6 methylprednisolone $4 \mathrm{mg} /$ day, nifedipine $20 \mathrm{mg} /$ day and acetylsalicylic acid $100 \mathrm{mg} /$ day. It improved the general and osteoarticular symptoms and the Raynaud's phenomenon, slowing down the progression of cutaneous involvement. Finally the lung picture appeared stable in follow-up CT after 2 years from the onset of symptoms.

\section{CONCLUSIONS}

Silicosis tends to be overlooked by rheumatologists as a possible cause for autoimmune disease. Its identification becomes important especially in case of patients with SSc, since the lung involvement is an important aspect in the prognostic outcome.

Therefore, it is essential to define the prevailing mechanism of the lung injury to identify the most effective treatment for these patients.

\section{REFERENCES}

1. Erasmus LD. Scleroderma in goldminers on the Witwatersrand with particular reference to pulmonary manifestations. S Afr J Lab Clin Med. 1957; 3: 209-31.

2. Rodnan GP, Benedek TG, Medsger TA, Cammarata RJ. The association of progressive systemic sclerosis (scleroderma) with coal workers' pneumoconiosis and other forms of silicosis. Ann Intern Med. 1967; 66: 323-34.

3. McCormic ZD, Khuder SS, Aryal BK, Ames AL, Khuder SA. Occupational silica exposure as a risk factor for scleroderma: a meta-analysis. Int Arch Occup Environ Health. 2010; 83: 763-9.

4. Koeger AC, Lang T, Alcaix D, Milleron B, Rozenberg S, Chaibi P, et al. Silica-associated connective tissue disease. A study of 24 cases. Medicine (Baltimore). 1995; 74: 221-37.

5. Makol A, Reilly MJ, Rosenman KD. Prevalence of connective tissue disease in silicosis (1985-2006) - a report from the state of Michi- gan surveillance system for silicosis. Am J Ind Med. 2011; 54: 255-62.

6. Nagata S. Fas and Fas ligand: a death factor and its receptor. Adv Immunol. 1994; 57: 12944.

7. Peng SL. Fas (CD95)-related apoptosis and rheumatoid arthritis. Rheumatology (Oxford). 2006; 45: 26-30.

8. Otsuki T, Tomokuni A, Sakaguchi H, Hyodoh F, Kusaka M, Ueki A. Reduced expression of the inhibitory genes for Fas-mediated apoptosis in silicosis patients. J Occup Health. 2000; 42: $163-8$.

9. Guo ZQ, Otsuki T, Shimizu T, Tachiyama S, Sakaguchi H, Isozaki Y, et al. Reduced expression of survivin gene in PBMC from silicosis patients. Kawasaki Med J. 2001; 27: 75-81.

10. Wu P, Miura Y, Hyodoh F, Nishimura Y, Hatayama $\mathrm{T}$, Hatada $\mathrm{S}$, et al. Reduced function of CD4+25+ regulatory $\mathrm{T}$ cell fraction in silicosis patients. Int J Immunopathol Pharmacol. 2006; 19: 357-68.

11. Otsuki T, Maeda M, Murakami S, Hayashi H, Miura Y, Kusaka M, et al. Immunological effects of silica and asbestos. Cell Mol Immunol. 2007; 4: 261-8.

12. Zaghi G, Koga F, Nisihara RM, Skare TL, Handar A, Rosa Utiyama SR, et al. Autoantibodies in silicosis patients and in silica-exposed individuals. Rheumatology International. 2010; 30: 1071-5.

13. McHugh NJ, Whyte J, Harvey G, Haustein UF. Antitopoisomerasi I antibodies in silicaassociated systemic sclerosis. A model for autoimmunity. Arthritis Rheum. 1994; 37: 1198205.

14. Rustin MH, Bull HA, Ziegler V, Mehlhorn J, Haustein UF, Maddison PJ, et al. Silica-associated systemic sclerosis is clinically, serologically and immunologically indistinguishable from idiopatic systemic sclerosis. Br J Dermatol. 1990; 123: 725-34.

15. Cointrel C, Tillie-Leblond I, Lamblin C, Furon D, Tonnel AB, Wallaer B. Erasmus syndrome: clinical, tomographic, respiratory function and bronchoalveolar lavage characteristics. Rev Mal Respir. 1997; 14: 21-6.

16. Ajlani H, Meddeb N, Sahli H, Sellami S. Erasmus syndrome: case report. Rev Pneumol Clin. 2009; 65: 16-22.

17. Boulanger M, Bienvenu B, Marquignon MF, Letourneux M, Clin B. Systemic sclerosis and occupational exposures: about a case in a driller-powderman. Rev Med Interne. 2014; pii: S0248-8663(14)00626-2. [Epub ahead of print].

18. Chaouch N, Mjid M, Zarrouk M, Rouhou SC, Ammous I, Hantous S. Erasmus' syndrome with pseudo-tumour masses. Rev Mal Respir. 2011; 28: 924-7.

19. Ben Abdelghani K, Fazaa A, Souabni L, Za- 
kraoui L. Association of pulmonary silicosis and systemic sclerosis. BMJ Case Rep. 2015; pii: bcr2013202509.

20. Sherson D, Maltbaek N, Olsen O. Small opacities among dental laboratory technicians in Copenhagen. Br J Ind Med. 1988; 45: 320-4.

21. Leclerc P, Fiessinger JN, Capron F, Ameille J, Rochemaure J. Erasmus syndrome in a dental technician. Importance of the prevention of occupational hazards. Ann Med Interne (Paris). 1983 ; $134: 653-5$.

22. de Miranda AA, Nascimento AC, Peixoto IL, Scrignoli JA, Cardoso Mdo S, Ribeiro SL. Erasmus syndrome: silicosis and systemic sclerosis. Rev Bras Reumatol. 2013; 53: 3103.

23. Devulder B, Plouvier B, Martin JC, Lenoir L. The association scleroderma-silicosis or Eras- mus' syndrome. Nouv Presse Med. 1977; 6: 2877-9.

24. van den Hoogen F, Khanna D, Fransen J, Johnson SR, Baron M, Tyndall A, et al. 2013 classification criteria for systemic sclerosis: an American College of Rheumatology/European League against Rheumatism collaborative iniziative. Arthritis Rheum. 2013; 65: 2737-47.

25. White B, Moore WC, Wigley FM, Xiao HQ, Wise RA. Cyclophosphamide is associated with pulmonary function and survival benefit in patients with scleroderma and alveolitis. Ann Intern Med. 2000; 132: 947-54.

26. Bussone G, Mouthon L. Interstitial lung disease in systemic sclerosis. Autoimmun Rev. 2011; 10: 248-55.

27. Leung CC, Yu IT, Chen W. Silicosis. Lancet. 2012; 379: 2008-18. 